Planta Med 2014; 80 - WS12

DOI: $10.1055 / \mathrm{s}-0034-1394553$

\title{
Unveiling Sambucus nigra L. berries terpenic metabolomics by comprehensive two dimensional gas chromatography: On the route of bioactive compounds
}

\author{
AC Salvador 1르, AJD Silvestre 르, SM Rocha 1
}

- $\quad$ 'QOPNA, Department of Chemistry, University of Aveiro, Campus Universitário de Santiago, 3810 - 193 Aveiro, Portugal

- $\quad{ }^{2}$ CICECO, Department of Chemistry, University of Aveiro, Campus Universitário de Santiago, 3810 - 193 Aveiro, Portugal

Metabolomics has expanded on the study of medicinal plants namely on the secondary metabolites plant profiling, that are linked to diverse health benefits on humans, arising as a suitable approach to evaluate biochemical-mediated responses. Elderberries have been used for generations in folk medicine, with several health benefits being reported [1]. The presence of secondary metabolites that are naturally present on elderberries, namely volatile terpenic [2], and phenolic [3] compounds could explain some of the reported biological activities, and being also responsible for some aspects of elderberries quality [4]. Therefore, the characterization of these compounds is of the major interest to understand elderberries biological properties and quality. However, the inherent complexity of the natural matrices requires analytical tools that solve their complexity, exceeding the capacity of the conventional single separation processes, and thus, limiting a reliable MS identification. Comprehensive two-dimensional gas chromatography arises as a possible solution employing two orthogonal mechanisms to separate the constituents of the sample within a single analysis, thus, increasing the peak capacity. In the present study, the volatile terpenic (mono and sesquiterpenic derivatives) and norisoprenoid compounds from the three major elderberries cultivars ('Sabugueira', 'Sabugueiro' and 'Bastardeira') grown in Portugal (5 ripening stages, collected during two consecutive harvesting years), were characterized by comprehensive two-dimensional gas chromatography. A total of 90 constituents were identified, being distributed over monoterpenic (47), sesquiterpenic (24), and norisoprenoid (19) families. From these, 65 are reported for the first time as $S$. nigra L. berries components. Harvesting season significantly affected the berries composition $(\mathrm{p}<0.05)$, however, ripening stage and cultivars effects should also be taken into consideration.

\begin{abstract}
Acknowledgements: Funding is acknowledged from the European Regional Development Fund (FEDER) through the Competitive Factors Thematic Operational Program (COMPETE) and from the Foundation for Science and Technology (FCT), Portugal, for funding the Research Units QOPNA (Research Unit 62/94 QOPNA, under projects PEstC/QUI/UI0062/2013; FCOMP-01 - 0124-FEDER-037296), and CICECO (project Pest-C/CTM/LA0011/2013; FCOMP-01 - 0124-FEDER-037271). The authors also thank RégieFrutas - Cooperativa Agrícola do Távora/Varosa for the samples supply, and Eng. César Pereira, Eng. Nuno Dias, and Eng. João Trindade for their technical support. A. Salvador thanks the FCT for the PhD grant (SFRH/BD/73778/2010).
\end{abstract}

Keywords: Metabolomics, elderberries, terpenic compounds, norisoprenoids, SPME, GC $\times$ GC-ToFMS

\section{References:}

[1] Vlachojannis JE, Cameron M et al. (2010) Phyther Res 24: 1 - 8.

[2] Jensen K, Christensen LP et al. (2001)J Sci Food Agric 81: 237 - 244.

[3] Veberic R, Jakopic J, et al. (2009) Food Chem 114: 511 - 515.

[4] Kaack K, Christensen LP et al. (2005) Eur Food Res Technol 221: 244 - 254. 